\title{
Oxygen-evolving complex of Photosystem II: An analysis of second- shell residues and hydrogen-bonding networks
}

\author{
Leslie Vogt, David J. Vinyard, Sahr Khan, Gary W. Brudvig* \\ Department of Chemistry, Yale University, New Haven, Connecticut 06520-8107, United States \\ ${ }^{*}$ Corresponding Author: \\ gary.brudvig@yale.edu \\ Phone: (203) 432-5202 \\ Yale University \\ Department of Chemistry \\ PO Box 208107 \\ New Haven, CT 06520-8107
}

The oxygen-evolving complex (OEC) is a $\mathrm{Mn}_{4} \mathrm{O}_{5} \mathrm{Ca}$ cluster embedded in the Photosystem II (PSII) protein complex. As the site of water oxidation, the OEC is connected to the lumen by channels that conduct water, oxygen, and/or protons during the catalytic cycle. The hydrogen-bond networks found in these channels also serve to stabilize the oxidized intermediates, known as the $S$ states. We review recent developments in characterizing these networks via protein mutations, molecular inhibitors, and computational modeling. Based on these results, we highlight regions of the PSII protein in which changes have indirect effects on the $S_{1}, S_{2}$, and $S_{3}$ oxidation states of the OEC while still allowing photosynthetic activity. 


\section{Introduction}

Photosystem II (PSII) is a light-driven water-plastoquinone (PQ) oxidoreductase. This large $(700 \mathrm{kDa})$ homodimeric complex is embedded in the thylakoid membrane of cyanobacteria, algae, and higher plants. As revealed by structural studies in cyanobacteria [1], each monomer is composed of 17 transmembrane subunits and three extrinsic subunits; PsbO, PsbU, and PsbV (PsbO, PsbP, and PsbQ in higher plants) that bind to the lumenal PSIl surface. The PSII reaction center core consists of the subunits D1, D2, CP43, CP47, and cytochrome $b_{559}$ ( $\alpha$ and $\beta$ subunits). The active site of water oxidation is the oxygen-evolving complex (OEC) where $\mathrm{O}_{2}$ is produced and protons are released into the lumen (eq 1). The OEC consists of $\mathrm{ann}_{4} \mathrm{CaO}_{5}$ inorganic cluster ligated by waters and amino-acid side chains (Figure 1A) [1].

$$
2 \mathrm{H}_{2} \mathrm{O}+4 \mathrm{hv} \rightarrow \mathrm{O}_{2}+4 \mathrm{H}^{+}{ }_{\text {lumen }}+4 \mathrm{e}^{-}
$$

In PSII, primary charge separation occurs at the reaction center chlorophyll-a electron donor, $\mathrm{P}_{680}$. The excited state, $\mathrm{P}_{680}{ }^{*}$, donates an electron to pheophytin, which in turn reduces the primary $P Q$ electron acceptor, $Q_{A}$. $Q_{A}{ }^{-}$reduces the secondary $P Q$ electron acceptor, $Q_{B}$. After receiving two electrons and two protons, plastoquinol $\left(Q_{B} \mathrm{H}_{2}\right)$ diffuses out of its binding pocket and is replaced by an oxidized PQ molecule from the membrane-soluble PQ pool. At the donor side of PSII, the hole remaining in $\mathrm{P}_{680}{ }^{+}$is filled by a redox-active tyrosine, $\mathrm{Y}_{\mathrm{Z}}$, which is subsequently reduced by the OEC.

At the OEC, $\mathrm{O}_{2}$ is produced via a four-step stochastic mechanism in which each intermediate is produced following excitation of $\mathrm{P}_{680}$. Dark-adapted PSII can undergo single turnovers using short saturating flashes of visible light. Joliot found that $\mathrm{O}_{2}$ was generated in high yield after the third flash and every fourth flash thereafter [2]. Using these data, Kok and coworkers developed a model, now known as the Kok cycle, in which the OEC cycles through four light-generated intermediates known as $S$ states [3]. As shown in Figure 1B, dark-adapted PSIl poised in $S_{1}$ will be converted to $S_{4}$ following three flashes of light. Upon its formation, $S_{4}$ spontaneously releases $\mathrm{O}_{2}$ and reforms $\mathrm{S}_{0}$.

While the chemistry of OEC turnover has received much attention, a mechanism of substrate water binding and $\mathrm{O}-\mathrm{O}$ bond formation has yet to be generally agreed upon. Herein, we focus on the hydrogen-bond networks in the second-shell protein environment around the OEC that efficiently transport protons and $\mathrm{O}_{2}$ out of the active site, while simultaneously allowing water access to the OEC. The naturally occurring residues that ligate the OEC (Figure 1A) are required for efficient photoassembly and $\mathrm{O}_{2}$ evolution (reviewed in Ref. [4]). Protein mutants 
have also determined that $Y_{Z}$ is D1-Y161 and shares a hydrogen bond with D1-H190 (reviewed in Ref. [5]). Water oxidation is not possible, or at best diminished, when any of these first-shell residues are mutated. Second-shell residues also play a key role in proton transport and tuning the properties of the OEC. In this review, we focus on the non-ligating protein environment of PSII within $15 \AA$ of the OEC, in which mutations and channel perturbations have substantial effects on the hydrogen-bond networks.

\section{Channels in Photosystem II}

A number of water-filled channels in PSII protein structures can form long-range hydrogen-bonded networks. One series of connected voids in PSII crystal structures (named "i" [8], "back" [9], or A1/A2 [10]) was originally proposed to be a channel for water or $\mathrm{O}_{2}$ transport. This channel has been shown via molecular dynamics to be impermeable to water [11], with a barrier of $\sim 22 \mathrm{kcal} / \mathrm{mol}$ at the bottleneck composed of D1-L91, CP43-F292, and CP43-F358 [12]. This leaves the "narrow," "broad," and "large" channel systems as options for water entry from the lumen to the OEC (Figure 2, with nomenclature from $[9,13,14]$ ). The narrow channel connects to D1-D61 and the O4 $\mu$-oxo ligand of the OEC and has a calculated $9 \mathrm{kcal} / \mathrm{mol}$ barrier for water entry at the interface of D1-N338, D2-N350, and CP43-P334 [12]. Just beyond this barrier, the channel reaches the lumen at the PsbO and PsbU interface in cyanobacteria (Figure 2B). On the other side of D1-D61, the broad channel passes the D1-E65, D1-R334, D2-E312 bottleneck (12 kcal/mol water barrier [12]), exiting at the interface of the D2 and PsbO subunits (Figure 2B). This channel is postulated to serve as a proton-exit pathway $[15,16]$. Finally, the large channel, with its many branches, is a possible route for delivering substrate water to the OEC (Figure 2B). The calculated activation barrier of $10 \mathrm{kcal} / \mathrm{mol}$ for water molecules occurs at the junction of pathways near D1-E329 [12], a residue affected by the S-state transitions [17]. The many studies that introduce point mutations or external inhibitors take advantage of these channels to modify the electrostatic or hydrogen-bonding environment surrounding the OEC.

\section{Water substrate exchange}

The exchange of ${ }^{18} \mathrm{O}$-labeled bulk water with substrate waters of the OEC is a direct experimental probe of the hydrogen-bonding networks involved in water transport [18]. The two substrate waters required for the generation of one $\mathrm{O}_{2}$ molecule are referred to as the fast $\left(\mathrm{W}_{\mathrm{f}}\right)$ and slow $\left(W_{s}\right)$ waters based on their relative rates of exchange. Both $W_{f}$ and $W_{s}$ exchange rates have an inverse $\mathrm{H} / \mathrm{D}$ isotope effect of 0.73 and 0.94 , respectively [19]. Replacing $\mathrm{H}_{2} \mathrm{O}$ with $\mathrm{D}_{2} \mathrm{O}$ 
strongly perturbs the hydrogen-bonding network in the case of $W_{f}$ but hardly effects $W_{s}$, leading to the possibility that either the two substrates are delivered to the OEC via different water channels or there are multiple microscopic H/D isotope effects that can enhance or suppress the measured isotope effects of the two waters asymmetrically.

Mutations of the second-sphere amino acids around the OEC affect the ${ }^{18} \mathrm{O}$ water exchange rates, highlighting their role in substrate water transport. The D1-D61N mutation slows down both $W_{s}$ and $W_{f}$ exchange rates by 3 and 6.5 times, respectively [20]. This is especially surprising because mutations of some of the direct ligands to the OEC, such as D1-D170 and D1-E189, have less dramatic effects [18] indicating that hydrogen-bonding networks play important roles in water exchange kinetics.

A chloride ion, situated in the broad channel $6 \AA$ away from the OEC, also regulates substrate water exchange. ${ }^{18} \mathrm{O}$ water exchange kinetics in PSIl substituted with $\mathrm{Sr}^{2+} / \mathrm{Br}^{-}$and $\mathrm{Sr}^{2+} / \mathrm{I}^{-}$in place of $\mathrm{Ca}^{2+} / \mathrm{Cl}^{-}$have shown that exchange of $\mathrm{W}_{\mathrm{f}}$ is 1.5 times slower and $\mathrm{W}_{\mathrm{s}}$ is 9.5 times faster in the $\mathrm{S}_{3}$ state of PSII [21]. In contrast, in PSII substituted with only $\mathrm{Sr}^{2+}, \mathrm{W}_{\mathrm{f}}$ is unaffected while the $W_{s}$ exchange rate increased by a factor of 3-4 [22]. The difference in the water exchange rates of $\mathrm{Sr}^{2+}$ substituted and $\mathrm{Sr}^{2+} / \mathrm{Br}^{-}$or $\mathrm{Sr}^{2+} / l^{-}$substituted PSIl can be attributed to changes in the hydrogen-bond network around the anion in the broad channel.

\section{$Y_{Z}$ electron transfer}

Natural variations in the amino-acid sequence of D1 have been identified, mainly in cyanobacteria that express different D1 isoforms when grown under different conditions. While many of the alternate residues affect photoprotection and electron transfer [23], there are some substitutions in the region around the OEC that affect $Y_{Z}$ electron transfer and progression through the Kok cycle. The inclusion of the psbA2-encoded D1 protein of Thermosynechococcus elongatus hinders the $S_{2}$-to- $S_{3}$ transition [24], due to changes in the hydrogen-bond network around D1-P173M [25]. Site-directed mutation of D1-P173G in Synechocystis sp. PCC 6803 also shows decreased electron transfer from $Y_{Z}$ to $P_{680}$ and only $62 \%$ of wild-type $\mathrm{O}_{2}$ evolution [26]. Point mutations based on D1 protein variants in Synechocystis sp. PCC 6803 show that the D1-F186L mutation results in only 70\% of wild-type $\mathrm{O}_{2}$ evolution, while D1-P162S has no observable effect [27]. Tellingly, both residues are next to D1-Y161, but only the backbone of residue D1-186 provides a hydrogen bond to the water network around the OEC (see Figure 3B).

\section{Equilibrium between $S_{2}$ isomers}


$\mathrm{S}_{2}$, generally agreed to be $\mathrm{Mn}^{3+}\left(\mathrm{Mn}^{4+}\right)_{3}$, is the most accessible EPR-active state and is present as two isomers [28]. The $S=1 / 2$ spin state is characterized by a multiline EPR signal at $g=2$, while the $S=5 / 2$ spin state shows a broad EPR signal at $g \approx 4.1$. As recently reviewed by Pokhrel and Brudvig, the specific hydrogen-bonding network around the OEC strongly affects which $\mathrm{S}_{2}$ isomer is favored [29]. In PSII samples from higher plants with sucrose as a cryoprotectant, both isomers are present, but for wild-type cyanobacteria, only the $S=1 / 2 S_{2}$ state is observed [29]. However, for point mutants in Synechocystis sp. PCC 6803, the $S=5 / 2$ signal was detected in mutants D2-K317R [30], D1-A344G, D1-A344D, and D1-A344N [31]. PSIl containing the D2-K317R mutation is still dependent on $\mathrm{Cl}^{-}(\sim 6 \AA$ from $\mathrm{Mn} 4$ [6]), but has lower steady-state $\mathrm{O}_{2}$-evolution activity and less efficient $\mathrm{S}$-state turnover [30]. Presumably, the presence of D2-R317 not only decreases proton-release efficiency in the broad channel, but also propagates changes in the hydrogen-bonding environment directly surrounding the OEC. Mutants at D1-A344 likely affect the OEC environment more directly as the C-terminus of the D1 peptide that ligates $\mathrm{Ca}$ and $\mathrm{Mn} 2$ (Figure 1A) [6] following post-translational cleavage [32]. Changing the side chain of A344 is not likely to change OEC ligation, but would influence the surrounding hydrogen-bond network, which in turn may affect the relative populations of the two $\mathrm{S}_{2}$ isomers.

The absence of the $S=5 / 2 S_{2}$ isomer in wild-type cyanobacteria is not understood, so we analyzed the D1 sequences of cyanobacteria, algae, and plants for any differences that might account for the change in $\mathrm{S}_{2}$ isomer populations. Between cyanobacteria and higher plants, the most significant variation near hydrogen-bonding networks is located at D1-87. The change from asparagine in cyanobacteria and green algae to alanine in higher plants will modify the shape and flexibility of the narrow channel, while also removing a hydrogen-bonding residue. We postulate that altering this hydrogen-bond network modulates the relative energy of the two $S_{2}$ isomers (Figure $3 D$ ).

\section{Formation of the $S_{3}$ state}

Because the $S_{1}$-to- $S_{2}$ transition does not involve proton release, changes in the hydrogen-bonding network around the OEC should not cause large effects until the $S_{2}$-to- $S_{3}$ transition (Figure 1B). The $\mathrm{S}_{3}$ state, $\left(\mathrm{Mn}^{4+}\right)_{4}$, has recently been characterized by multidimensional EPR with each Mn center having octahedral coordination [33]. This implies that an additional ligand is incorporated during the $S_{2}$-to- $S_{3}$ transition to convert five-coordinate $\mathrm{Mn}(\mathrm{III})$ to six-coordinate Mn(IV), although it remains to be determined whether this occurs at $\mathrm{Mn} 1$ or $\mathrm{Mn} 4$. In contrast to the local OEC changes observed for the $\mathrm{S}_{1}$-to- $\mathrm{S}_{2}$ transition in state- 
resolved X-ray diffraction studies [34,35], protein motion in response to the formation of $S_{3}$ is observable at $5.5 \AA$ resolution [36]. The rearrangement of protein in the $S_{3}$ state must be accompanied by an adjustment of the hydrogen-bond network since many waters surrounding the OEC are stabilized by hydrogen bonds to backbone atoms.

\section{Proton-release pathways}

All S-state transitions except $S_{1}$ to $S_{2}$ involve proton release to the lumen (Figure 1B) as determined by $\mathrm{pH}$ dependence, $\mathrm{H} / \mathrm{D}$ isotope effects [37], and photoacoustic spectroscopy [38]. The $S_{3}$-to- $S_{0}$ transition occurs with a half-life of 1-2 ms [39] and is generally agreed to be the rate-limiting step of OEC turnover. While the OEC has a hypothetical maximum turnover frequency of $\geq 500 \mathrm{~s}^{-1}$, the observed turnover rate of PSII is $25-88 \mathrm{O}_{2} \mathrm{~s}^{-1}$ (reviewed in Ref. [40]). This kinetic limitation of OEC turnover is often controlled by PQ exchange, but can also be limited by proton release to the lumen [41].

The broad channel is postulated to be a proton-release pathway and is lined by a number of hydrogen-bonding residues (Figure 3B). This water-filled channel includes the area around D1-N181 and D1-V185 and extends past a chloride ion, D1-D61, and D2-K317 before reaching residues on the lumenal surface. The calculated $\mathrm{pK}_{\mathrm{a}}$ values for residues along the channel increase in the direction of the lumen, favoring proton transport away from the OEC [15]. Although the D1-E65, D1-R334, and D2-E312 residues form a $12 \mathrm{kcal} / \mathrm{mol}$ barrier for water transport [12], the two glutamate residues can share a proton and are postulated to be a "proton antenna" [16]. Experimental characterization shows that mutations D1-E65A [17], D2-E312A [17], D1-R334A [42], D1-D61A [43], and D2-K317A [30] or D2-K317R [44] affect carboxylate and amide FTIR features in similar ways. Based on FTIR data from other mutants, the hydrogen-bond network involved in proton release may even extend to D1-E329 [17] or D1-Q165 [42]. The side chain of D1-D61 clearly plays a role in stabilizing waters as the FTIR feature at $3663 \mathrm{~cm}^{-1}$ in the $\mathrm{O}-\mathrm{H}$ stretching region for weakly hydrogen-bonded waters is eliminated by the D1-D61A mutant [43]. X-ray exposure results in $67 \%$ of the broad channel residues showing oxidative modification due to generation of reactive oxygen species (ROS) from waters in the channel [45].

\section{$\mathrm{O}_{2}$-release pathways}

It remains unclear whether there is a dedicated route to facilitate the transport of $\mathrm{O}_{2}$ away from the OEC. In standard spinach PSII preparations, oxidative modification of protein residues, which may be due to ROS chemistry, occurs only along the narrow channel (i.e. at 
CP43: E354, T355, M356, and R357) [46] (Figure 3D). Derivatization of PSIl crystals with $\mathrm{Kr}$ resulted in two $\mathrm{Kr}$ atoms bound in the large channel system suggested to be an $\mathrm{O}_{2}$-exit pathway [10]. Subsequent computational simulations of $\mathrm{O}_{2}$ diffusion show that the $\mathrm{O}_{2}$ molecules generally follow the water pathways, with likely exit paths overlapping portions of either the large or broad channels $[47,48]$. Intriguingly, the D1-E329 residue that hinders water diffusion in the large channel poses no barrier for $\mathrm{O}_{2}$ diffusion [47] (Figure 3C), providing a likely mechanism for PSII to control water access to the OEC but facilitate the exit of $\mathrm{O}_{2}$.

Experimental determination of $\mathrm{O}_{2}$-release kinetics is also useful to highlight possible $\mathrm{O}_{2}$-exit pathways. For example, mutation of the $\mathrm{D} 2-\mathrm{K} 317$ residue in the broad channel results in slower $\mathrm{O}_{2}$ release [30]. Also near the OEC, the D1-V185N mutant has the slowest measured $\mathrm{O}_{2}$ release kinetics of any mutant [26]. It remains possible that these mutations also affect the chemical mechanism of $\mathrm{O}-\mathrm{O}$ bond formation or the efficiency of proton release through the hydrogen-bond network rather than directly affecting $\mathrm{O}_{2}$-diffusion kinetics. Therefore, it would be interesting to explore whether mutations along the large channel would affect $\mathrm{O}_{2}$ release, although the branched structure of the large channel system may require careful choice of mutants to study. For example, in proteins created by random mutagenesis, both the single mutant D1-N322I and the double mutant D1-I326F/D1-F328S showed impaired growth in high light, but the triple mutant is phototolerant [49]. The slow-growing D1-L341P mutant [50], however, is an interesting candidate for further characterization.

\section{Conclusions}

The hydrogen-bond networks surrounding the OEC on the lumenal side of the PSII protein serve to stabilize charged intermediates and regulate the structure of the channels that allow transport of water, protons, and $\mathrm{O}_{2}$. While many residues lining the broad channel have been characterized, there are opportunities to probe the water network in the narrow and large channels. Amino-acid residues along these channels can affect the hydrogen-bond networks directly via side-chain interactions or by perturbing the location of the protein backbone. Furthermore, comparing the species- and growth condition-dependent differences in these regions of the PSII complex will help elucidate which residues are important for regulating the complicated water-oxidation chemistry.

\section{Acknowledgements}

Funded by the U.S. Department of Energy, Office of Science, Office of Basic Energy Sciences (Grant No. DE-FG02-05ER14646). 


\section{References}

- of special interest

•- of outstanding interest

•• 1. Suga M, Akita F, Hirata K, Ueno G, Murakami H, Nakajima Y, Shimizu T, Yamashita K, Yamamoto $\mathrm{M}$, Ago H, et al.: Native structure of photosystem II at $1.95 \AA$ A resolution viewed by femtosecond X-ray pulses. Nature 2014, doi:10.1038/nature13991.

This X-ray free electron laser study presents the first high-resolution "radiation-damage-free" structure of the OEC.

2. Joliot $P$, Barbieri $G$, Chabaud R: Un nouveau modele des centres photochimiques du systeme II. Photochem Photobiol 1969, 10:309-329.

3. Kok B, Forbush B, McGloin M: Cooperation of charges in photosynthetic $\mathrm{O}_{2}$ evolution-I. A linear four step mechanism. Photochem Photobiol 1970, 11:457-475.

4. Debus RJ: Protein Ligation of the Photosynthetic Oxygen-Evolving Center. Coord Chem Rev 2008, 252:244-258.

5. Diner BA: Amino acid residues involved in the coordination and assembly of the manganese cluster of photosystem II. Proton-coupled electron transport of the redox-active tyrosines and its relationship to water oxidation. Biochim Biophys Acta 2001, 1503:147-163.

6. Umena Y, Kawakami K, Shen J-R, Kamiya N: Crystal structure of oxygen-evolving Photosystem II at a resolution of 1.9 angstrom. Nature 2011, 473:55-60.

7. Pal R, Negre CFA, Vogt L, Pokhrel R, Ertem MZ, Brudvig GW, Batista VS: $\mathbf{S}_{0}-$ State Model of the Oxygen-Evolving Complex of Photosystem II. Biochemistry 2013, 52:7703-7706.

8. Murray JW, Barber J: Structural characteristics of channels and pathways in photosystem II including the identification of an oxygen channel. J Struct Biol 2007, 159:228-237.

9. Ho FM, Styring S: Access channels and methanol binding site to the CaMn ${ }_{4}$ cluster in Photosystem II based on solvent accessibility simulations, with implications for substrate water access. Biochim Biophys Acta 2008, 1777:140-153.

10. Gabdulkhakov A, Guskov A, Broser M, Kern J, Muh F, Saenger W, Zouni A: Probing the accessibility of the $\mathrm{Mn}_{4} \mathrm{Ca}$ cluster in photosystem II: channels calculation, noble gas derivatization, and cocrystallization with DMSO. Structure 2009, 17:1223-1234.

11. Vassiliev S, Comte P, Mahboob A, Bruce D: Tracking the flow of water through photosystem II using molecular dynamics and streamline tracing. Biochemistry 2010, 49:1873-1881.

- 12. Vassiliev S, Zaraiskaya T, Bruce D: Exploring the energetics of water permeation in photosystem II by multiple steered molecular dynamics simulations. Biochim Biophys Acta 2012, 1817:1671-1678.

The authors locate energetic barriers for water diffusion using steered molecular dynamics simulations to move water molecules along possible transport pathways.

13. Ho FM: Structural and mechanistic investigations of photosystem II through computational methods. Biochim Biophys Acta 2012, 1817:106-120.

14. Linke K, Ho FM: Water in Photosystem II: structural, functional and mechanistic considerations. Biochim Biophys Acta 2014, 1837:14-32.

15. Ishikita H, Saenger W, Loll B, Biesiadka J, Knapp EW: Energetics of a possible proton exit pathway for water oxidation in photosystem II. Biochemistry 2006, 45:2063-2071.

16. Bondar AN, Dau H: Extended protein/water H-bond networks in photosynthetic water oxidation. Biochim Biophys Acta 2012, 1817:1177-1190.

17. Service RJ, Hillier W, Debus RJ: Evidence from FTIR difference spectroscopy of an extensive network of hydrogen bonds near the oxygen-evolving $\mathrm{Mn}_{4} \mathrm{Ca}$ cluster of photosystem II involving D1-Glu65, D2-Glu312, and D1-Glu329. Biochemistry 2010, 49:6655-6669.

18. Cox N, Messinger J: Reflections on substrate water and dioxygen formation. Biochimica $E t$ Biophysica Acta-Bioenergetics 2013, 1827:1020-1030.

19. Hillier W, Wydrzynski T: Substrate water interactions within the Photosystem II oxygen evolving complex. PCCP 2004, 6:4882-4889. 
20. Singh S, Debus RJ, Wydrzynski T, Hillier W: Investigation of substrate water interactions at the high-affinity Mn site in the photosystem II oxygen-evolving complex. Philosophical Transactions of the Royal Society B-Biological Sciences 2008, 363:1229-1234.

21. Nilsson H, Rappaport F, Boussac A, Messinger J: Substrate-water exchange in photosystem II is arrested before dioxygen formation. Nat Commun 2014, 5:4305.

22. Hendry G, Wydrzynski T: 0-18 isotope exchange measurements reveal that calcium is involved in the binding of one substrate-water molecule to the oxygen-evolving complex in photosystem II. Biochemistry 2003, 42:6209-6217.

23. Vinyard DJ, Gimpel J, Ananyev GM, Cornejo MA, Golden SS, Mayfield SP, Dismukes GC: Natural variants of photosystem II subunit D1 tune photochemical fitness to solar intensity. J Biol Chem 2013, 288:5451-5462.

24. Sugiura M, Ogami S, Kusumi M, Un S, Rappaport F, Boussac A: Environment of TyrZ in photosystem II from Thermosynechococcus elongatus in which PsbA2 is the D1 protein. $J$ Biol Chem 2012, 287:13336-13347.

- 25. Sugiura M, Ozaki Y, Nakamura M, Cox N, Rappaport F, Boussac A: The D1-173 amino acid is a structural determinant of the critical interaction between D1-Tyr161 (TyrZ) and D1-His190 in Photosystem II. Biochim Biophys Acta 2014, 1837:1922-1931.

Construction of point mutants at D1-173 allows the authors to demonstrate that this residue affects $Y_{Z}$ electron transfer during the $\mathrm{S}_{2}$-to- $\mathrm{S}_{3}$ transition and accounts for an observed difference in natural D1 protein variants in Thermosynechococcus elongatus.

-• 26. Dilbeck PL, Bao H, Neveu CL, Burnap RL: Perturbing the water cavity surrounding the manganese cluster by mutating the residue D1-valine 185 has a strong effect on the water oxidation mechanism of photosystem II. Biochemistry 2013, 52:6824-6833.

By constructing point mutations for second-shell residues, the authors determine that D1-V185 is important for accumulation of PSII, stabilization of charge separation, and $\mathrm{O}_{2}$ release. The effects of other second-shell mutants that can affect the hydrogen-bonding network are reported in the Supporting Information.

27. Wiklund R, Salih GF, Maenpaa P, Jansson C: Engineering of the protein environment around the redox-active TyrZ in photosystem II. The role of F186 and P162 in the D1 protein of Synechocystis 6803. Eur J Biochem 2001, 268:5356-5364.

28. Pantazis DA, Ames W, Cox N, Lubitz W, Neese F: Two Interconvertible Structures that Explain the Spectroscopic Properties of the Oxygen-Evolving Complex of Photosystem II in the $\mathbf{S}_{2}$ State. Angew Chem Int Ed 2012, 51:9935-9940.

29. Pokhrel R, Brudvig GW: Oxygen-evolving complex of photosystem II: correlating structure with spectroscopy. Phys Chem Chem Phys 2014, 16:11812-11821.

30. Pokhrel R, Service RJ, Debus RJ, Brudvig GW: Mutation of Lysine 317 in the D2 Subunit of Photosystem II Alters Chloride Binding and Proton Transport. Biochemistry 2013, 52:47584773.

31. Mizusawa N, Yamanari T, Kimura Y, Ishii A, Nakazawa S, Ono T-a: Changes in the Functional and Structural Properties of the Mn Cluster Induced by Replacing the Side Group of the CTerminus of the D1 Protein of Photosystem II. Biochemistry 2004, 43:14644-14652.

32. Nixon PJ, Trost JT, Diner BA: Role of the carboxy-terminus of polypeptide D1 in the assembly of a functional water-oxidizing manganese cluster in photosystem II of the cyanobacterium Synechocystis sp. PCC 6803:assembly requires a free carboxyl group at C-terminal position 344. Biochemistry 1992, 31:10859-10871.

33. Cox N, Retegan M, Neese F, Pantazis DA, Boussac A, Lubitz W: Photosynthesis. Electronic structure of the oxygen-evolving complex in photosystem II prior to O-O bond formation. Science 2014, 345:804-808.

34. Kern J, Alonso-Mori R, Tran R, Hattne J, Gildea RJ, Echols N, Glockner C, Hellmich J, Laksmono H, Sierra RG, et al.: Simultaneous femtosecond X-ray spectroscopy and diffraction of photosystem II at room temperature. Science 2013, 340:491-495.

35. Askerka M, Wang J, Brudvig GW, Batista VS: Structural Changes in the Oxygen-Evolving Complex of Photosystem II Induced by the $S_{1}$ to $S_{2}$ Transition: A Combined XRD and QM/MM Study. Biochemistry 2014, 53:6860-6862. 
36. Kupitz C, Basu S, Grotjohann I, Fromme R, Zatsepin NA, Rendek KN, Hunter MS, Shoeman RL, White TA, Wang D, et al.: Serial time-resolved crystallography of photosystem II using a femtosecond X-ray laser. Nature 2014, 513:261-265.

37. Renger G: Mechanism of light induced water splitting in Photosystem II of oxygen evolving photosynthetic organisms. Biochimica et Biophysica Acta (BBA) - Bioenergetics 2012, 1817:1164-1176.

-38. Klauss A, Haumann M, Dau H: Alternating electron and proton transfer steps in photosynthetic water oxidation. Proc Natl Acad Sci U S A 2012, 109:16035-16040.

The authors use volume changes due to electron and proton transfer to show when these alternating events occur during the Kok cycle.

39. Canaani O, Malkin S, Mauzerall D: Pulsed photoacoustic detection of flash-induced oxygen evolution from intact leaves and its oscillations. Proceedings of the National Academy of Sciences, USA 1988, 85:4725-4729.

40. Vinyard DJ, Ananyev GM, Charles Dismukes G: Photosystem II: The Reaction Center of Oxygenic Photosynthesis. Annu Rev Biochem 2013, 82:577-606.

41. Carrieri D, Ananyev G, Brown T, Dismukes GC: In vivo bicarbonate requirement for water oxidation by Photosystem II in the hypercarbonate-requiring cyanobacterium Arthrospira maxima. J Inorg Biochem 2007, 101:1865-1874.

42. Service RJ, Hillier W, Debus RJ: Network of hydrogen bonds near the oxygen-evolving $\mathrm{Mn}_{4} \mathrm{CaO}_{5}$ cluster of photosystem II probed with FTIR difference spectroscopy. Biochemistry 2014, 53:1001-1017.

43. Debus RJ: Evidence from FTIR difference spectroscopy that D1-Asp61 influences the water reactions of the oxygen-evolving $\mathrm{Mn}_{4} \mathrm{CaO}_{5}$ cluster of photosystem II. Biochemistry 2014, 53:2941-2955.

44. Suzuki H, Yu J, Kobayashi T, Nakanishi H, Nixon PJ, Noguchi T: Functional roles of D2-Lys317 and the interacting chloride ion in the water oxidation reaction of photosystem II as revealed by fourier transform infrared analysis. Biochemistry 2013, 52:4748-4757.

45. Frankel LK, Sallans L, Bellamy H, Goettert JS, Limbach PA, Bricker TM: Radiolytic mapping of solvent-contact surfaces in Photosystem II of higher plants: experimental identification of putative water channels within the photosystem. J Biol Chem 2013, 288:23565-23572.

- 46. Frankel LK, Sallans L, Limbach PA, Bricker TM: Identification of oxidized amino acid residues in the vicinity of the $\mathrm{Mn}_{4} \mathrm{CaO}_{5}$ cluster of Photosystem II: implications for the identification of oxygen channels within the Photosystem. Biochemistry 2012, 51:6371-6377.

Mass spectrometry is used to determine that amino acids from core PSII proteins are oxidized in spinach preparations. Importantly, several oxidized residues of CP43 are found along the narrow channel.

47. Vassiliev S, Zaraiskaya T, Bruce D: Molecular dynamics simulations reveal highly permeable oxygen exit channels shared with water uptake channels in photosystem II. Biochim Biophys Acta 2013, 1827:1148-1155.

48. Zaraiskaya T, Vassiliev S, Bruce D: Discovering oxygen channel topology in photosystem II using implicit ligand sampling and wavefront propagation. Journal of Computational Science 2014, 5:549-555.

49. Narusaka $\mathrm{Y}$, Narusaka $\mathrm{M}$, Satoh $\mathrm{K}$, Kobayashi $\mathrm{H}$ : In vitro random mutagenesis of the D1 protein of the photosystem II reaction center confers phototolerance on the cyanobacterium Synechocystis ps. PCC 6803. J Biol Chem 1999, 274:23270-23275.

50. Minda R, Joshi VP, Bhattacharjee SK: psbA gene family coding D1 proteins of photosystem II of cyanobacteria Synechocystis PCC 6803 is essential for DNA repair/protection. Current Science 2011, 100:58-68. 


\section{Figure Legends}

Figure 1. (A) Structure of the OEC and coordinating residues from the $1.9 \AA$ crystal structure [6] and computationally optimized for the $\mathrm{S}_{1}$ state [7] showing $\mathrm{Mn}$ (purple), $\mathrm{Ca}$ (orange) and $\mathrm{O}$ (red). All labeled side chains are from the D1 residue, except CP43-Glu354. (B) The Kok cycle. Starting from the darkstable $S_{1}$ state, electrons (blue) and protons (red) are released in an alternating pattern [3]. $\mathrm{O}_{2}$ is released upon formation of the $\mathrm{S}_{4}$ state.

Figure 2. (A) The water-filled channels around the OEC are shown in the context of the PSII monomer [6]. Subunits with residues that line the channels are highlighted in color: D1 and D2 in blues, antenna proteins in greens, and PsbO in purple. Water molecules shown in orange form the "narrow" $[9,13,14]$ channel (also known as E/F [10], or channel 2 [12]). The yellow surface shows the water network corresponding to the channels termed "broad" $[9,13,14]$, "iii" [8], D/C/G [10], or 1/3 [12]. In red is the branching network described as the "large channel system" [9,13,14], "ii" [8], B1/B2 [10], or 4a/4b [12]. (B) The water molecules in the $1.9 \AA$ crystal structure are shown as spheres within each colored-mesh surface for the channels shown in panel (A) [6]. The large channel (in red) also contains two glycerol molecules introduced during crystallization. The protein environment around each channel is depicted as colored ribbons (D1: dark blue, D2: light blue, CP43: light green).

Figure 3. (A) The channel systems detailed in Figure 1B are shown without the protein environment. In panels B-D, important side chains and hydrogen-bonding backbone atoms are shown in licorice with the protein displayed as ribbons (D1: dark blue, D2, light blue, CP43: light green, PsbU/PsbV: gray). The OEC atoms are shown as connected spheres with Mn (purple), Ca (orange), and O (red). Important residues and water molecules that are first-shell ligands to the OEC are labeled in bold. Transparent red surfaces highlight residues along each channel calculated to form a barrier to water transport [12]. (B) The water molecules that form the broad channel are shown outlined in a yellow mesh. The location of chloride ion in the $1.9 \AA$ crystal structure is shown as a green sphere [6]. (C) Many branches join to create the large channel (red mesh). Two glycerol molecules found in the crystal structure [6] are also included inside the mesh outline of the channel. (D) The narrow channel (orange mesh) extends past residues that show oxidative modification [47] and may link to another channel for water transport (channel X, not shown) found during molecular dynamics simulations [12]. 


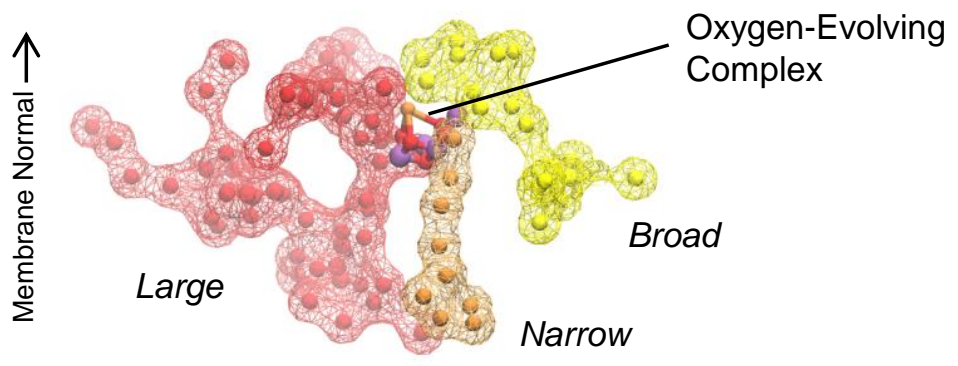


Figure 1
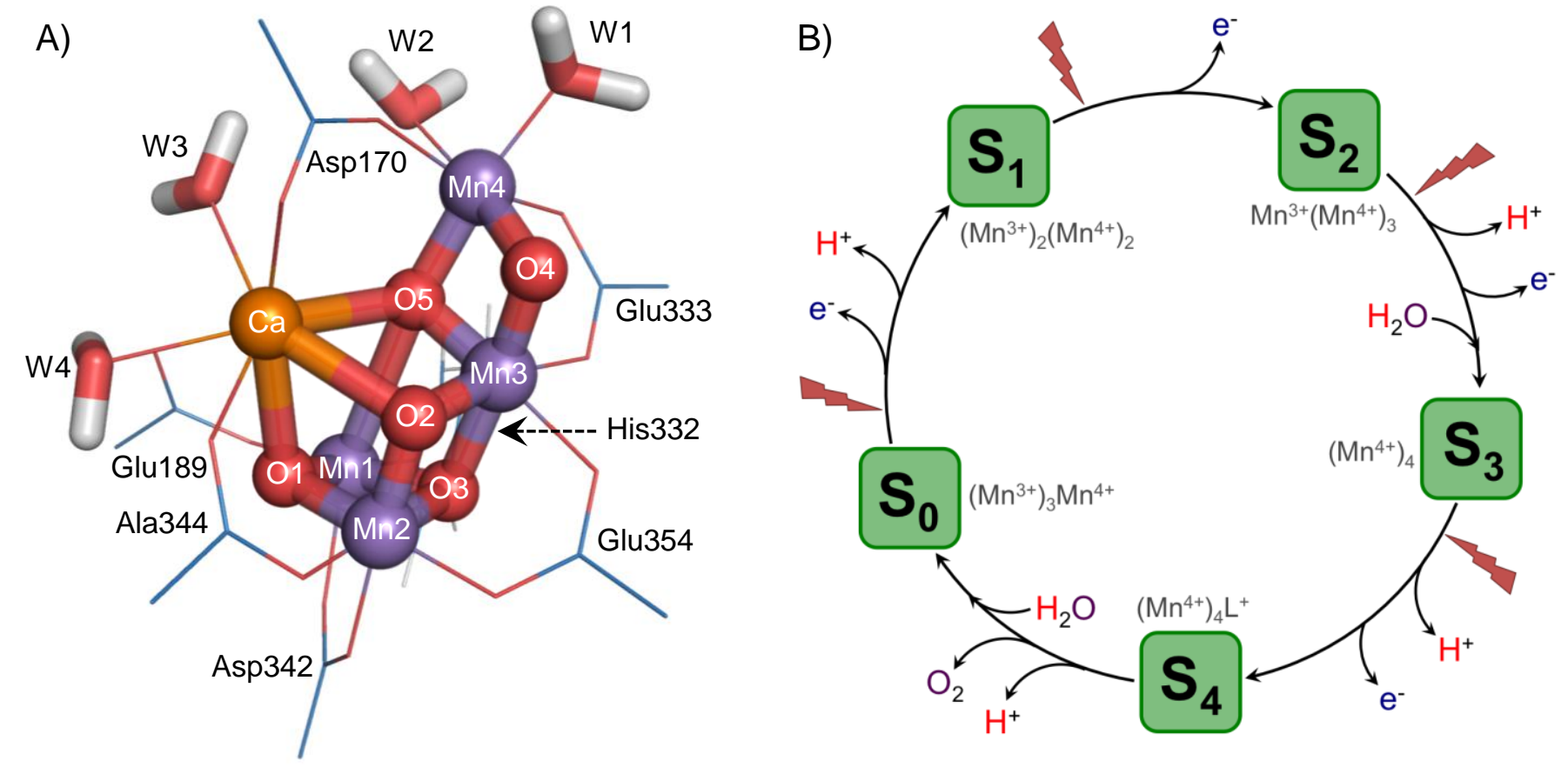
Figure 2

B)

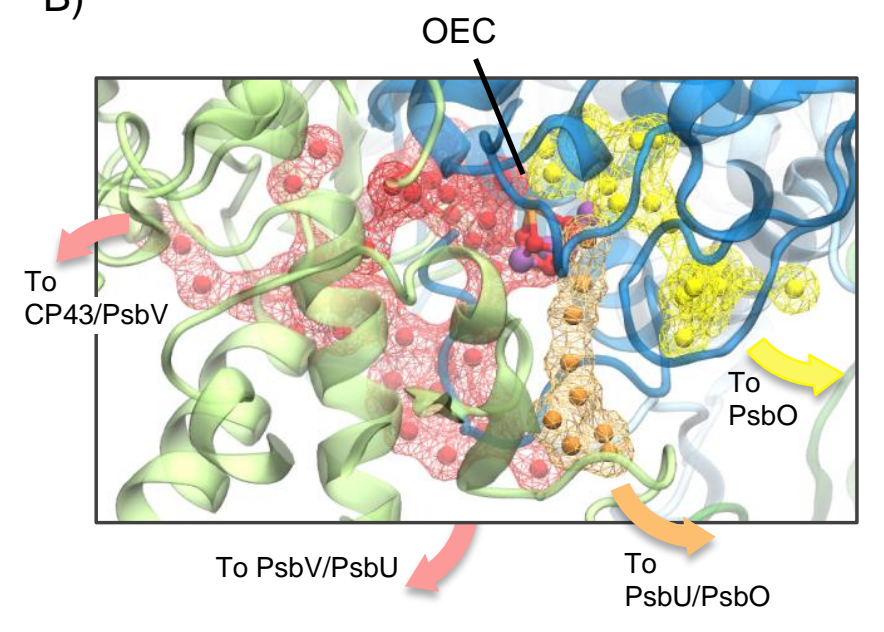

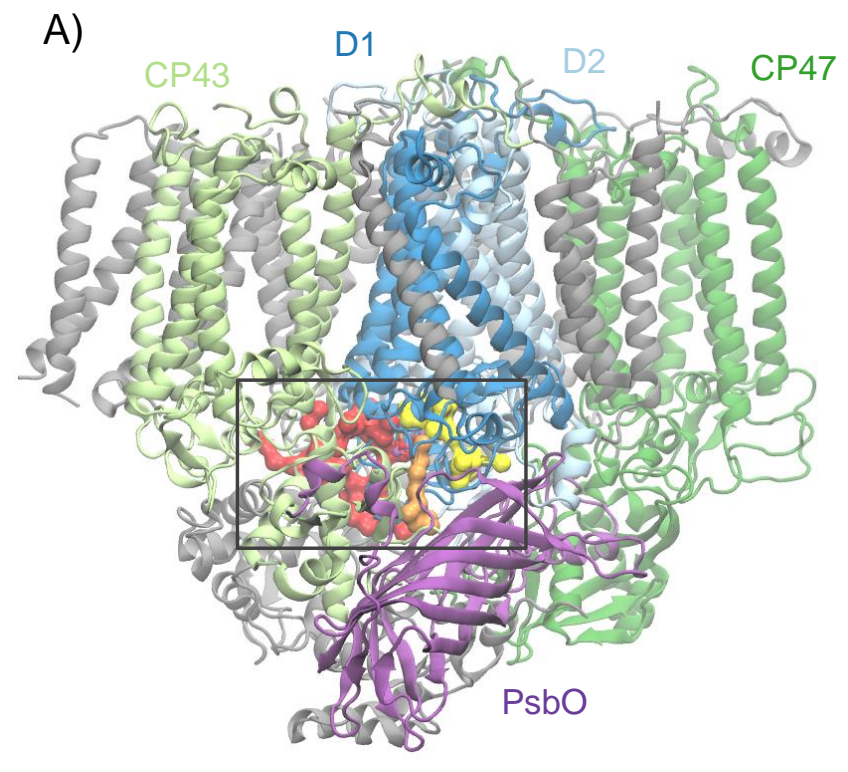

\section{A)}


Figure 3
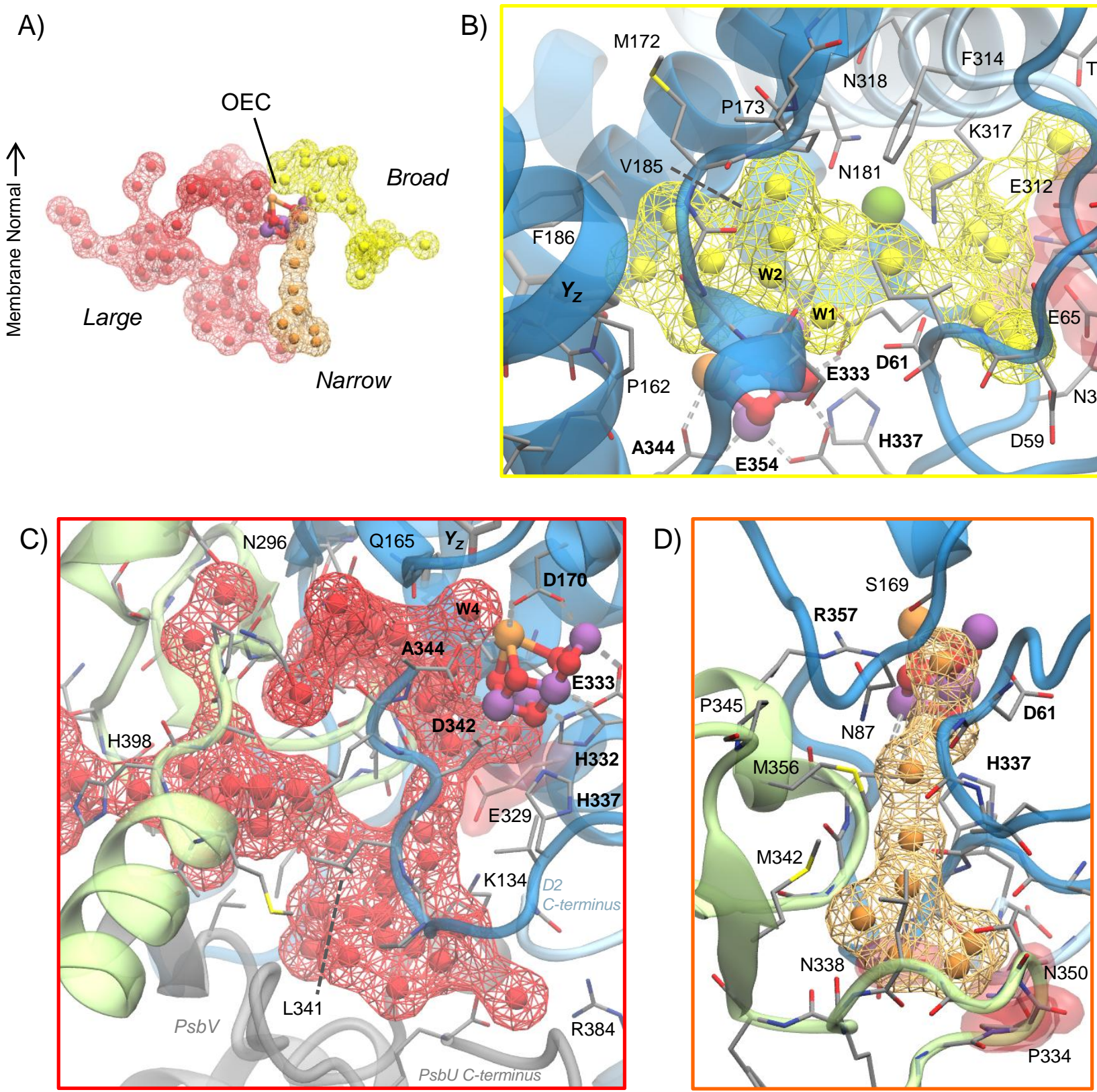